\title{
PROBLEMAS DE COBERTURA EM LOCALIZAÇÃO DE INSTALAÇÕES: UMA REVISÃO SISTEMÁTICA DA LITERATURA
}

\author{
Daniela Biccas Ferraz Matos \\ Pontifícia Universidade Católica do Rio de Janeiro - PUC - RJ \\ R. Marquês de São Vicente, 225 - Gávea, Rio de Janeiro - RJ, 22451-900 \\ danielabiccas@uol.com.br \\ Antonio Márcio Tavares Thomé \\ Pontifícia Universidade Católica do Rio de Janeiro - PUC - RJ \\ R. Marquês de São Vicente, 225 - Sala 952 - Gávea, Rio de Janeiro - RJ, 22451-900 \\ mt@puc-rio.br
}

\section{RESUMO}

A localização de instalações é uma área muito importante e complexa da logística, e um de seus principais problemas tange à cobertura. Devido à escassez da metodologia de revisão sistemática de literatura acerca dos problemas de cobertura em localizações de instalações após um trabalho publicado em 2012, ainda que haja crescimento do interesse na área acadêmica, este artigo visa fornecer uma revisão sistemática de literatura (RSL) sobre o tema. Essa revisão permite uma abordagem baseada em evidências que identifica, seleciona e analisa dados secundários, obtendo uma visão geral mais objetiva da pesquisa. Entre os artigos publicados a partir de 2011, foram inicialmente selecionados 190, e, após a aplicação da metodologia, restaram 86. Após essa etapa, eles foram caracterizados de acordo com a metodologia utilizada e temas abordados. Este texto fornece uma visão geral dos principais problemas de localização de instalação e por que adotar problemas de cobertura, e tem como objetivo demonstrar a importância dessa metodologia na elaboração de um artigo, bem como mostrar os temas em que estes tipos de problemas são mais aplicados e recomendar temas de futuras pesquisas.

Palavras-chave: Localização de instalações; cobertura; problemas de cobertura; revisão; revisão sistemática da literatura.

\begin{abstract}
The facility location is a very important and complex area of logistics, and one of its main problems is with coverage. Due to the scarcity of the systematic literature review methodology concerning coverage problems in facility locations, following a work published in 2012, even though there is growing interest in the academic field, this paper aims to provide a systematic literature review (RSL) about the theme. This review allows an evidence-based approach that identifies, selects, and analyses secondary data for a more objective overview of the research. Among the articles published since 2011, 190 of them were initially selected and after the implementation of the methodology, 86 artciles remained. After this stage, they were characterized according to the methodology used and the topics addressed. This text provides an overview of the main issues regarding facility
\end{abstract}


location and the reasons to adopt coverage issues, and aims to demonstrate the importance of this methodology in the elaboration of an article. As well as to display the topics in which these types of problems are recurring and recommend topics for future research.

Keywords: Facility location; Covering; Covering problem; Review; Systematic literature review.

\section{Como Citar:}

MATOS, Daniela Biccas Ferraz; THOMÉ, Antonio Márcio Tavares. Problemas de Cobertura em Localização de Instalações: Uma Revisão Sistemática da Literatura. In: SIMPÓSIO DE PESQUISA OPERACIONAL E LOGÍSTICA DA MARINHA, 19., 2019, Rio de Janeiro, RJ. Anais [...]. Rio de Janeiro: Centro de Análises de Sistemas Navais, 2019.

\section{INTRODUÇÃO}

Segundo Farahani e Hekmatfar [1], a localização de instalações consiste em localizar um conjunto de instalações para minimizar o custo de satisfazer algum conjunto de demanda com relação a algum conjunto de restrições. Esta é uma área muito importante e complexa da logística. Por ser um tema bastante desenvolvido dentro de Pesquisa Operacional, pesquisadores desenvolveram diversos trabalhos ao longo do tempo que abordam modelos ou problemas relacionados a essa área. Snyder [2] diz que as decisões de localização de instalações são custosas e difíceis de reverter, por isso devem ser feitas de forma a serem mais eficientes e eficazes possíveis, com o objetivo de evitar gastos desnecessários e desperdício de tempo.

Uma característica relevante de localização de instalações é a possibilidade de aplicações em outras áreas, considerando os custos e distância do ponto de demanda. Lu e Bostel [3] utilizaram localização de instalações em um sistema de logística reversa. Balcik e Beamon [4] desenvolveram um modelo que determina o número e a localização dos centros de distribuição em uma rede de ajuda e a quantidade de suprimentos a serem estocados nos centros de distribuição de forma a atender as pessoas necessitadas. Melo, Nickel e Saldanhada-Gama [5] realizaram uma revisão compreensiva da literatura em localização de instalações e cadeia de suprimentos. Farahani et al. [6], por sua vez, afirmam que uma das principais aplicações de localizações de instalações são os problemas de cobertura, cuja aplicabilidade em casos reais, principalmente na área de emergência e serviços, faz com que seja muito importante na logística.

Conforme Owen e Daskin [7], uma instalação é considerada coberta quando uma distância ou tempo máximo, definido por ela, for atendido dentro de um tempo específico. Os problemas de localização de cobertura podem abranger serviços de emergência, tais como ambulâncias e corpo de bombeiros. Eles são classificados em dois modelos: problema de cobertura (Set Covering Problem) e problema de localização de máxima cobertura (Maximal Covering Location Problem, MCLP) [6; 8]. O primeiro foi introduzido inicialmente por Toregas et al. [9], cujo objetivo é minimizar o custo da localização da instalação de forma que um nível de cobertura seja obtido [10]. O segundo é abordado por Church e Revelle [11], que almejam maximizar a demanda coberta dentro de uma distância de serviço aceitável localizando um número pré-definido de instalações [7].

Uma área que possui grandes problemas de cobertura é a saúde, pois é primordial alocar de forma efetiva e eficaz todas as instalações de atendimento de emergência, por ser 
um campo essencial em nossas vidas. Acerca desse ponto, Li et al. [12] abordam diversos modelos de cobertura e técnicas de otimização para localização e planejamento de instalações de resposta a emergências, e McLay [13] aplicou um problema de localização de cobertura máxima esperada com dois tipos de servidores (Maximum Expected Coverage Location Problem with Two Types of Servers), com o objetivo de otimizar a localização e uso das unidades médicas de forma a melhorar a sobrevivência do paciente.

Rousseau, Manning e Denyer [14] dizem que devem ser abordadas as perguntas de pesquisa claramente definidas, de forma rigorosa, com o objetivo de serem bem especificadas, informativas e claramente formuladas, para evitar-se ambiguidade. De forma a garantir isso, as seguintes perguntas de pesquisa foram formuladas:

- PP-1: Quais métodos de pesquisa estão sendo utilizados?

- PP-2: Quais temas de pesquisa estão sendo abordados?

Farahani et al. [6] realizaram uma revisão da literatura na qual abordam modelos, soluções e aplicações relacionados a problemas de cobertura após o trabalho apresentado por Schilling, Jayaraman e Barkhi [8]. Este artigo, por seu turno, tem como objetivo fornecer uma RSL de problemas de cobertura em localização de instalações, de forma a mostrar os temas em que são mais aplicados, após a revisão de Farahani et al. [6]. O trabalho é organizado como a seguir: o próximo capítulo descreve a metodologia adotada. O capítulo 3 relata e discute os resultados da revisão. No último capítulo, será feito a conclusão e sugestões de pesquisas futuras neste assunto.

\section{METODOLOGIA}

A RSL foi escolhida como método de pesquisa para este trabalho, pois localiza estudos existentes, seleciona e avalia contribuições, além de analisar e sintetizar dados de forma a alcançar conclusões claras sobre o que é conhecido e desconhecido, conforme Denyer e Tranfield [15]. Thomé, Scavarda e Scavarda [16] definiram RSL como uma importante pesquisa em si e não somente uma revisão de escritores anteriores. O principal objetivo dessa metodologia é resumir o corpo acumulado de conhecimento sobre as relações de interesse e determinar onde existem lacunas, conforme mostra Gligor e Holcomb [17].

De forma a reduzir o viés durante a pesquisa e assegurar a replicabilidade, este estudo seguiu as diretrizes definidas em Thomé, Scavarda e Scavarda [16]. Elas consistem em um passo a passo composto de oito etapas para conduzir uma RSL em Gerenciamento de Operações: (1) planejamento e formulação do problema; (2) pesquisando a literatura; (3) coleta de dados; (4) avaliação da qualidade; (5) análise e síntese de dados; (6) interpretação; (7) apresentação dos resultados; e (8) atualização da revisão.

\subsection{Pesquisando a Literatura}

Primeiramente, houve a seleção da base de dados a ser utilizada e as perguntas de pesquisa, que já foram definidas na introdução. Para este trabalho, foi selecionado a base SCOPUS, pois, segundo Farahani et al. [6], é uma das maiores bases de dados de título, resumo e palavra-chave a checar trabalhos relacionados e para ver a tendência dessa pesquisa ao longo do tempo. Mongeon e Paul-Hus [18] afirmam que a base SCOPUS tem um número maior de periódicos exclusivos do que Web of Science em todos os campos, pelo fato de cobrir muito mais jornais. A pesquisa foi limitada a partir de fevereiro de 2011, pois este artigo tem como objetivo fornecer uma RSL de problemas de cobertura em localização de instalações após a revisão de Farahani et al. [6], a qual abrange até 2018.

Após a leitura de diversos artigos sobre o assunto (e.g., [1], [2], [6], [7]), as seguintes palavras-chave foram definidas: ( ( TITLE-ABS-KEY ( covering AND location )) AND ( ("covering problem" ) ) ) AND ( ( ("facility location" ) ) AND ("covering 
location problem" ) ) AND ( PUBYEAR > 2010$)$.

\subsection{Coleta de Dados e Avaliação da Qualidade}

Como todos os trabalhos encontrados são de periódicos revisados por pares, pode-se considerar que essa seleção minimiza as chances de incluir avaliações de baixa qualidade e não confiáveis na análise [16]. A primeira pesquisa resultou em 190 documentos. Os métodos de seleção e exclusão consistiram em: artigos com texto completo disponível unicamente foram selecionados, e artigos que não estavam diretamente relacionados com o tema foram excluídos.

Após aplicar esses métodos e realizar uma referência cruzada de todas as citações e bibliografias, foi possível identificar potenciais contribuições que podem ter sido perdidas. Finalmente, todos os artigos foram lidos na sua íntegra, resultando em um banco de dados contendo 86 documentos acadêmicos publicados em jornais revisados por pares.

\subsection{ANÁliSE DE DAdOS, SíNTESE E INTERPRETAÇÃO}

Todos os documentos selecionados foram classificados de acordo com a sua metodologia, que são, segundo Seuring e Müller [19]: artigos conceituais e teóricos; estudos de casos; surveys; trabalhos de modelagem; e revisões de literatura. Eles são posteriormente classificados com relação ao foco de cada estudo e suas principais questões abordadas. Os artigos com mais de um tipo de metodologia foram classificados de acordo com a principal. Após essa análise, foi possível identificar os principais temas relacionados a problemas de cobertura em localização de instalações:

- Serviços de emergência, em termos de ter a maior cobertura possível nas áreas da saúde, natureza e desastres naturais.

- Design dos nós, em termos do projeto/dimensionamento dos nós de demanda e da abrangência do raio de cobertura.

- Cliente, em termos de considerar a preferência do cliente ou de ser o foco na modelagem dos problemas.

- Centros de distribuição, em termos de localizar e/ou alocar a demanda de centros de distribuições ou hubs.

Os principais métodos utilizados foram igualmente identificados:

- Combinação de algoritmos, em termos de combinar mais de um algoritmo de forma a resultar em um novo modelo.

- Sob efeito de incerteza, em termos de estar sujeito a incerteza, seja no tempo, na demanda, e verificar o seu efeito.

- Comparação entre problemas, em termos de comparar dois ou mais tipos de problemas de cobertura.

\section{RESULTADOS E DISCUSSÃO}

O Gráfico 1 demonstra as metodologias usadas desde 2011 até 2018. Nela, é possível ver que, apesar de survey ter sido listada, ela não foi utilizada. O ano com maior número de artigos publicados foi 2016, e as metodologias mais usadas foram modelagem e estudo de caso, sendo revisão a menos utilizada de todas.

O Quadro 1 relaciona os dez artigos mais citados na base SCOPUS - a qual será descrita nas subseções 3.1 e 3.2 -, de forma a resumir os temas mais abordados, juntamente com o método e sua metodologia. Ele apresenta artigos mais antigos, ou seja, aqueles que tiveram mais tempo para acumular citações. De forma a evitar viés, bem como apresentar os artigos dos anos mais recentes, serão representados os quatro artigos mais citados dos anos 
2017 e de 2018 no Quadro 2.

Gráfico 1 - Metodologias utilizadas pelos artigos

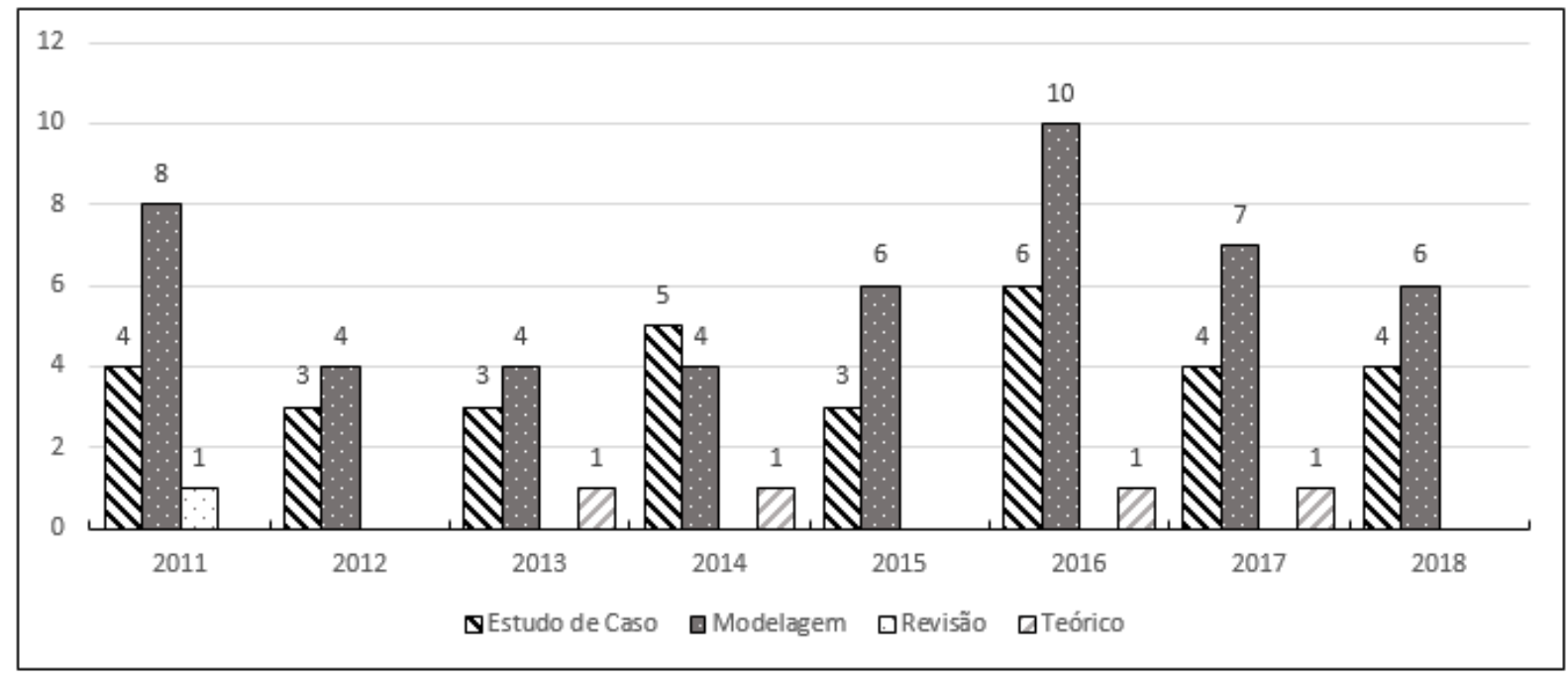

Elaboração dos autores.

Quadro 1 - Artigos mais citados na base SCOPUS

\begin{tabular}{|c|c|c|c|c|}
\hline Autor(es) & $\begin{array}{l}\text { Número de } \\
\text { citações } \\
\text { (Nov./2018) }\end{array}$ & Metodologia & Tema & Método \\
\hline Li et al. [12] & 127 & Revisão & Serviços de Emergência & Comparação Entre Problemas \\
\hline $\begin{array}{l}\text { Murali, Ordóñez e Des- } \\
\text { souky [20] }\end{array}$ & 57 & Estudo de Caso & Serviços de Emergência & Sob Efeito de Incerteza \\
\hline $\begin{array}{l}\text { Mohammadi, Jolai e Rosta- } \\
\text { mi [21] }\end{array}$ & 52 & Modelagem & Centros de Distribuição & Combinação de Algoritmos \\
\hline $\begin{array}{l}\text { Mohammadi, Jolai e Ta- } \\
\text { vakkoli-Moghaddam [22] }\end{array}$ & 47 & Modelagem & Centros de Distribuição & Sob Efeito de Incerteza \\
\hline $\begin{array}{l}\text { Zarandi, Sisakht e Davari } \\
\text { [23] }\end{array}$ & 44 & Modelagem & Design dos Nós & Sob Efeito de Incerteza \\
\hline $\begin{array}{l}\text { Karimi e Bashiri [24] } \\
\text { Knight, Harper e Smith [25] } \\
\text { Davari, Zarandi e Hemmati }\end{array}$ & $\begin{array}{l}43 \\
37 \\
30\end{array}$ & $\begin{array}{l}\text { Modelagem } \\
\text { Modelagem } \\
\text { Modelagem }\end{array}$ & $\begin{array}{l}\text { Centros de Distribuição } \\
\text { Serviços de Emergência } \\
\text { Design dos Nós }\end{array}$ & $\begin{array}{l}\text { Combinação de Algoritmos } \\
\text { Combinação de Algoritmos } \\
\text { Sob Efeito de Incerteza }\end{array}$ \\
\hline $\begin{array}{l}\text { [26] } \\
\text { He, Kuo e Wu [27] } \\
\text { Chanta, Mayorga e McLay }\end{array}$ & $\begin{array}{l}29 \\
24\end{array}$ & $\begin{array}{l}\text { Estudo de Caso } \\
\text { Estudo de Caso }\end{array}$ & $\begin{array}{l}\text { Serviços de Emergência } \\
\text { Serviços de Emergência }\end{array}$ & $\begin{array}{l}\text { Comparação Entre Problemas } \\
\text { Combinação de Algoritmos }\end{array}$ \\
\hline 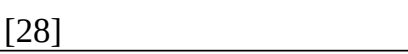 & & & & \\
\hline
\end{tabular}

Elaboração dos autores.

Quadro 2 - Artigos mais citados na base SCOPUS dos anos 2017 e 2018

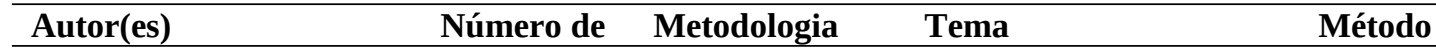




\begin{tabular}{|c|c|c|c|c|}
\hline & $\begin{array}{l}\text { citações } \\
\text { (Nov./2018) }\end{array}$ & & & \\
\hline Paul, Lunday e Nurre [29] & 8 & Estudo de Caso & Serviços de Emergência & Combinação de Algoritmos \\
\hline Zhang, Peng e Li [30] & 6 & Estudo de Caso & Serviços de Emergência & Sob Efeito de Incerteza \\
\hline Jayalakshm e Singh [31] & 5 & Modelagem & Design dos Nós & Combinação de Algoritmos \\
\hline Díaz et al. [32] & 3 & Modelagem & Cliente & Combinação de Algoritmos \\
\hline Akbari, Eiselt e Pelot [33] & 1 & Estudo de Caso & Serviços de Emergência & Comparação Entre Problemas \\
\hline $\begin{array}{l}\text { Bagherinejad e Shoeib } \\
\text { [34] }\end{array}$ & 1 & Modelagem & Design dos Nós & Combinação de Algoritmos \\
\hline $\begin{array}{l}\text { Madani, Nookabadi e He- } \\
\text { jazi [35] }\end{array}$ & 1 & Estudo de Caso & Centros de Distribuição & Combinação de Algoritmos \\
\hline Smith et al. [36] & 1 & Estudo de Caso & Serviço de Emergência & Combinação de Algoritmos \\
\hline
\end{tabular}
Elaboração dos autores.

\subsection{QUAIS MÉTOdOS DE PESQUISA ESTÃo SENDO UTILIZADOS?}

O Gráfico 2 mostra que o método mais utilizado foi combinação de algoritmos, e o Gráfico 3, a quantidade de artigos que utilizam cada método por metodologia. Analisandoos, verifica-se que comparação entre problemas foi o mais abordado nas metodologias teórica e revisão, enquanto nos outros dois, os mais abordados foram combinação de algoritmos.

Gráfico 2 - Métodos utilizados pelos artigos

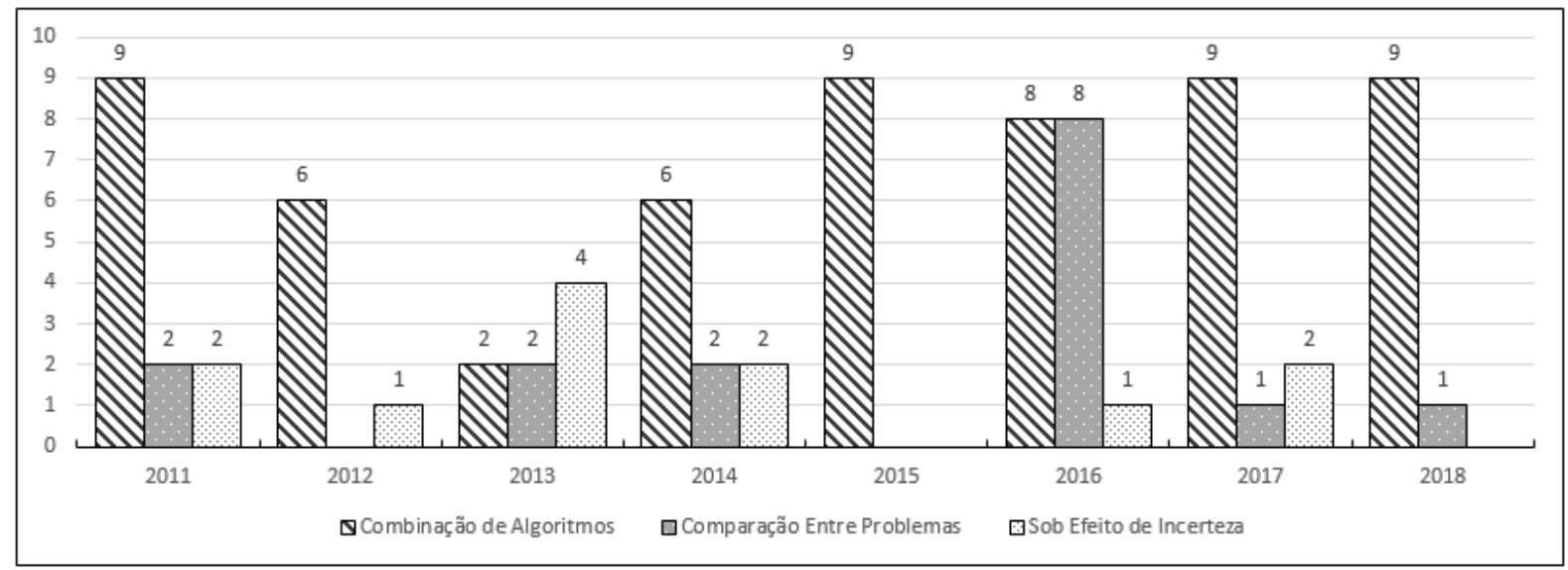

Elaboração dos autores.

Gráfico 3 - Métodos utilizados por cada metodologia 


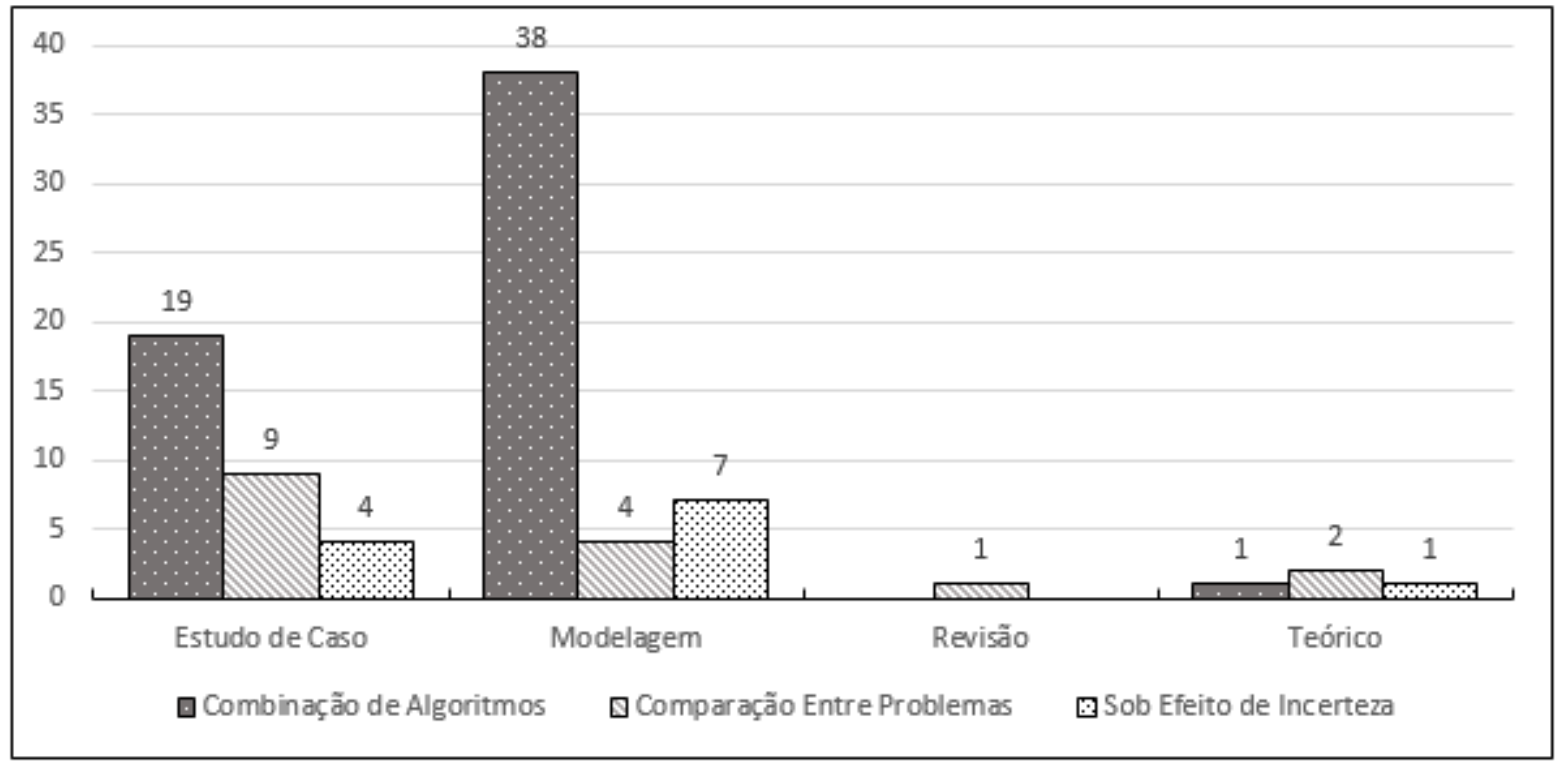

Elaboração dos autores.

\subsubsection{Combinação de Algoritmos}

Muitas vezes, um problema pode ser tão complexo, que necessita da combinação de mais de um algoritmo, de forma a ser possível achar uma solução para ele. Mohammadi, Jolai e Rostami [21] utilizam um algoritmo competitivo imperialista (Imperialist Competitive Algorithm, ICA), uma meta-heurística para problemas de otimização contínua. Esses autores, ao proporem um novo método de assimilação e usarem a função de cruzamento do algoritmo genético, levam a uma solução mais próxima do ótimo. Após estas alterações, o modelo é aplicado a uma otimização discreta.

Karimi e Bashiri [24] aplicam duas heurísticas nos quatro modelos de problemas de cobertura de hub: conjunto de hub e cobertura máxima, com alocação única e múltipla. A primeira calcula o custo fixo de estabelecer hubs nos nós de forma a minimizar o custo. A segunda calcula os pesos que fluem na rede estruturada, com o objetivo de maximizar o fluxo.

Knight, Harper e Smith [25] abordam uma versão estendida estocástica do Modelo de Localização de Sobrevivência Esperado Máximo para Pacientes Heterogêneos, cujo objetivo é retornar à probabilidade de sobrevivência, incorporando várias classes de pacientes (através de múltiplas classes de função de sobrevivência) no modelo original. Posteriormente, é utilizada a teoria de filas, de forma a permitir a modelagem do congestionamento e utilização de cada estação de ambulância.

Chanta, Mayorga e McLay [28] propõem três modelos biobjetivos para localizar ambulâncias, de forma a reduzir a diferença de tempo de espera de atendimento entre as zonas. O primeiro consiste em sempre maximizar a cobertura esperada, e o segundo visa melhorar o atendimento na zona rural. Para isso, o último objetivo irá assumir três funções: minimizar a distância máxima entre cada zona de demanda descoberta e sua estação aberta mais próxima; minimizar o número de zonas de demanda rural descobertas; e minimizar o número de zonas de demanda descobertas.

Paul, Lunday e Nurre [29] formulam uma extensão hierárquica multiobjetiva do problema de cobertura máxima, buscando maximizar a cobertura da população dentro de uma janela de resposta rápida e, ao mesmo tempo, minimizar as modificações na estrutura existente. Além disso, estendem uma restrição do problema de cobertura condicional 
(conditional covering problem, CCP), na qual o conjunto de demandas e o conjunto de possíveis instalações eram iguais, de forma a reduzir o risco para os recursos de resposta a emergências.

Jayalakshmi e Singh [31] propõem um algoritmo híbrido de colônia artificial de abelhas (hybrid artificial bee colony algorithm), uma meta-heurística baseada na população, para o problema de localização de cobertura máxima cooperativa (cooperative maximum covering location problem, CMCLP) em uma rede.

Díaz et al. [32] formulam uma extensão do problema de localização de máxima cobertura como um problema de programação matemática de dois níveis. Posteriormente, propõem uma heurística de procedimento de busca adaptativa aleatória gananciosa (greedy randomized adaptive search procedure, GRASP) e uma heurística híbrida GRASP-Tabu para resolver este problema.

Bagherinejad e Shoeib [34] desenvolvem um modelo integrando o problema de localização de máxima cobertura capacitada modular e o problema de localização máxima de cobertura de multiperíodo (dinâmico). Além disso, a restrição de capacidade dinâmica, na qual a capacidade muda periodicamente, é considerada nas instalações. Posteriormente, utilizam o algoritmo genético (genetic algorithm, GA) e o algoritmo de abelha para a resolução deste problema.

Madani, Nookabadi e Hejazi [35] desenvolvem um problema de cobertura máxima p-hub biobjetivo confiável e de alocação única, cujos objetivos são maximizar os fluxos cobertos esperados e minimizar congestionamento. Sendo uma extensão do problema de localização de cobertura máxima p-hub ( $p$-hub maximal covering location problem, pHMCP), foi utilizado um algoritmo genético de ordenação não dominada (NSGA-II) para resolvê-lo.

Smith et al. [36] utilizam uma extensão das técnicas analíticas de localização clássica, na qual empregam uma abordagem de cobertura de conjuntos modificada, gerando um algoritmo capaz de permitir escolhas claramente justificadas ao se alocar a demanda a um tipo de laboratório específico.

\subsubsection{Sob Efeito de Incerteza}

Murali, Ordónñez e Dessouky [20] utilizam problemas de localização de instalações de forma a determinar pontos em uma grande cidade, onde a população iria para receber remédios ou atenção médica. Porém, a priori, o fornecimento em cada instalação depende de uma demanda desconhecida. Assim, modelam como um problema de localização de instalações capacitadas e usam restrições aleatórias para lidar com a incerteza da demanda.

Mohammadi, Jolai e Rostami [22] desenvolvem um modelo de transporte multiobjetivo e multimódulo estocástico para problemas de localização de cobertura hub sob efeito de incerteza. Segundo os autores, custos, demandas, distâncias, tempo e outros parâmetros do problema podem mudar devido a um ambiente incerto, gerando assim maior custo de investimento e um tempo maior de transporte na rede.

Zarandi, Sisakht e Davari [23] utilizam uma programação de metas interativas difusas para modelar um problema de rede de distribuição da cadeia de suprimentos de circuito fechado, pois uma solução ótima para todos os objetivos é considerada bem difícil. Após, um modelo de design de rede de distribuição multiobjetivo é desenvolvido.

Davari, Zarandi e Hemmati [26] consideram que o tempo entre viagens entre cada nó é considerado uma variável difusa, por não ter uma informação precisa deste tempo e ele ser difícil de estimar. Posteriormente, aplicam esta variável a um problema de localização de cobertura máxima difusa.

Zhang e Peng [30] utilizam os tempos de resposta e a demanda como variáveis incertas, pelas emergências e a gravidade do acidente serem imprevisíveis. Em seguida, o 
problema de localização de cobertura e o problema de localização de máxima cobertura são modelados em um ambiente incerto.

\subsubsection{Comparação Entre Problemas}

Li et al. [12] abordam e comparam diversos modelos matemáticos de cobertura aplicados no planejamento e localização de instalações de serviços de emergência, bem como as técnicas de otimização utilizadas para resolvê-los.

$\mathrm{He}$, Kuo e $\mathrm{Wu}$ [27] comparam os locais ótimos gerados das estações públicas de carregamento de veículos elétricos de três modelos de localização: modelo de cobertura ( $\mathrm{Set}$ Covering Model); modelo de localização de máxima cobertura (Maximal Covering Location Model); e modelo da p-mediana ( $P$-median Model). Dessa forma, eles obtiveram o modelo mais eficiente para a localização destas instalações em Pequim.

Akbari, Eiselt e Pelot [33] visam incorporar vários tipos de instalações dos recursos de busca e resgate marítimo em dois problemas de análise de localização: o problema de localização máxima de cobertura (MCLP); e problemas p-mediana. Logo após, comparam as soluções obtidas com relação a vários critérios de decisão.

\subsection{QUAIS TEMAS DE PESQUISA ESTÃO SENDO ABORDADOS?}

O Gráfico 4 mostra os temas mais abordados, e conclui-se que eles são: serviços de emergência e design de nós. O Gráfico 5, por sua vez, mostra a quantidade de artigos que abordam cada tema por metodologia. No estudo de caso, o mais abordado é serviços de emergência, enquanto em modelagem o mais utilizado foi design dos nós.

Gráfico 4 - Temas abordados pelos artigos

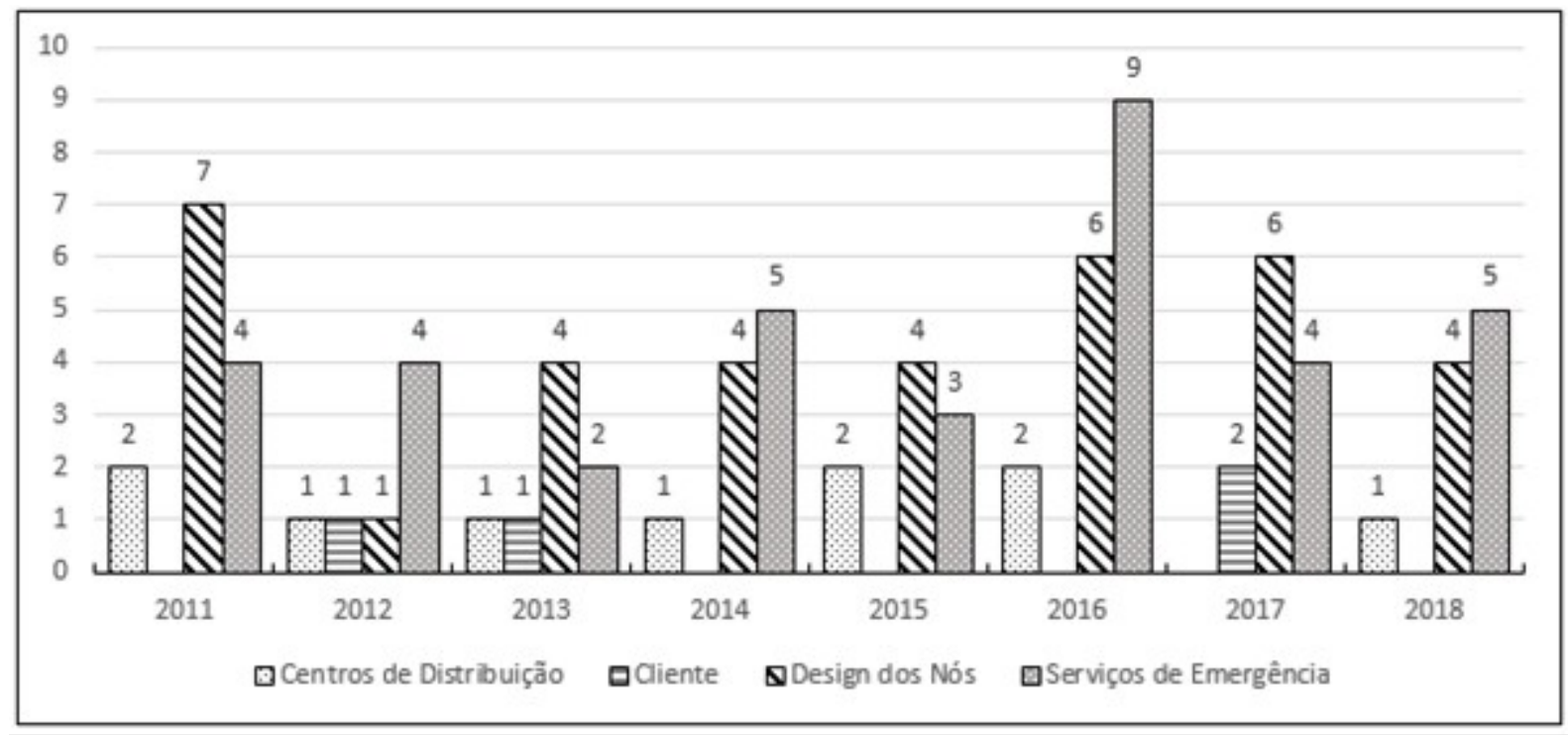

Elaboração dos autores. 
Gráfico 5 - Temas abordados por metodologia

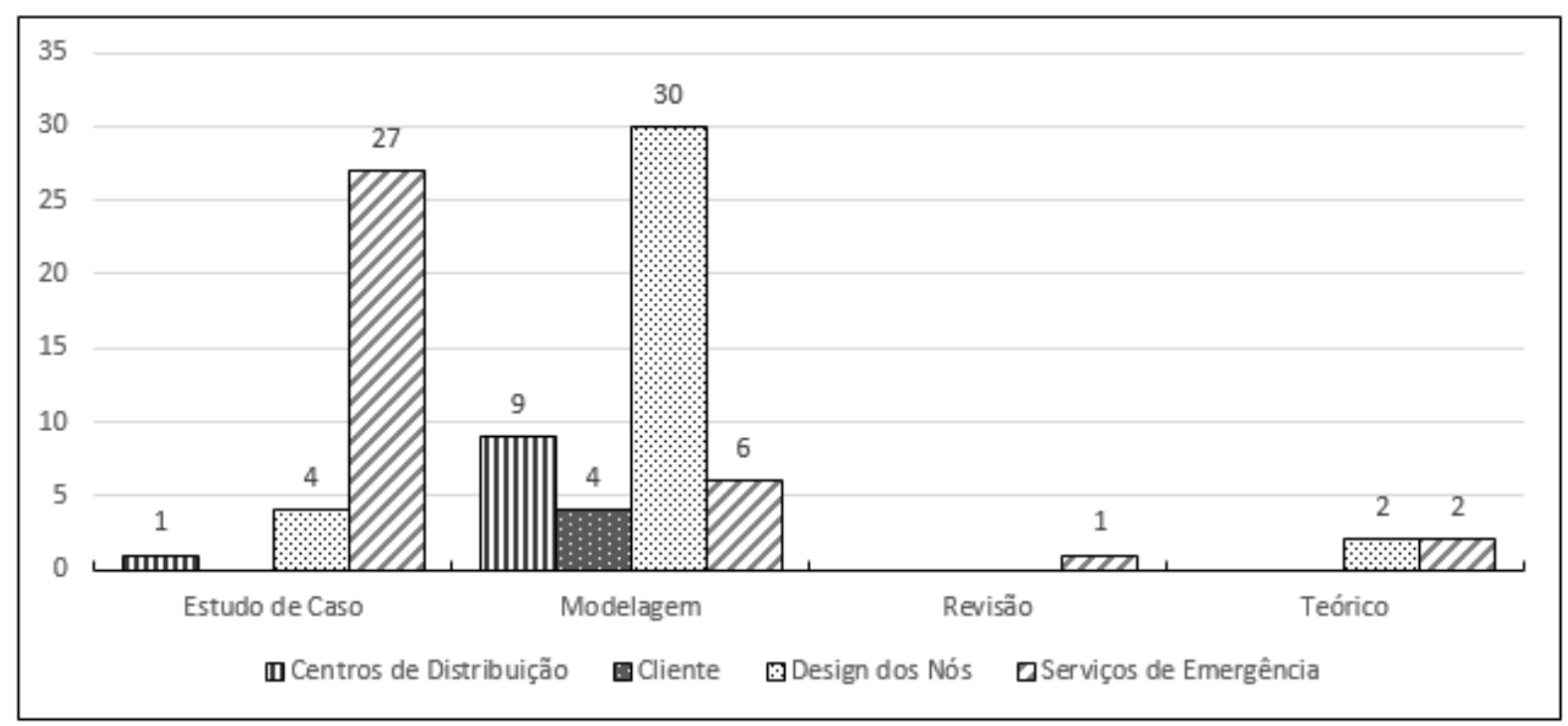

Elaboração dos autores.

\subsubsection{Serviços de Emergência}

Pouco tem sido discutido sobre os novos modelos de cobertura, como de alocação dinâmica, cobertura gradual, entre outros. Para suprir essa lacuna, Li et al. [12] revisam modelos de cobertura com ênfase em desenvolvimentos mais recentes e técnicas de otimização para planejamento e localização de instalação de emergência. Além disso, abordam os modelos de cobertura clássicos, bem como suas extensões.

Murali, Ordóñez e Dessouky [20] utilizam problemas de localização de instalações de forma a determinar pontos em uma grande cidade onde remédios devem ser distribuídos para a população no caso de um ataque bioterrorista. Levam em conta uma função dependente da distância e uma demanda incerta na modelagem, a fim de maximizar a cobertura. Posteriormente, aplica a um estudo de caso, em que localiza instalações durante uma emergência de grande escala de um ataque hipotético de antraz em Los Angeles.

Knight, Harper e Smith [25] utilizam um modelo de localização de um serviço médico de emergência (SEM), cujo objetivo é maximizar a probabilidade de sobrevivência de todas as classes múltiplas de clientes, de forma a resultar em modelos probabilísticos mais realistas para pacientes heterogêneos. Ele foi aplicado posteriormente a um estudo de caso na região.

He, Kuo e Wu [27] debatem sobre um estudo de caso em Pequim, de forma a localizar as estações públicas de carregamento dos veículos elétricos, cujo objetivo é incorporar as restrições de oferta e demanda dessas estações em modelos de localização de instalações. Com isso, fornecem políticas com uma análise compreensiva para melhor entender a eficiência desses modelos na localização das estações.

Chanta, Mayorga e McLay [28] analisam a diferença de atendimento médico de emergência entre as zonas rural e urbana. Muitos desses sistemas de atendimento visam maximizar a demanda, consequentemente indo para áreas mais populosas e gerando uma espera de atendimento maior em áreas rurais. Os autores visam reduzir esta diferença de tempo entre as zonas com três modelos propostos citados anteriormente.

Paul, Lunday e Nurre [29] analisam a eficácia das localizações ótimas de ativos regionais mantidos pelo Departamento de Defesa dos Estados Unidos para responder a emergências de grande escala. Visam, com o modelo e metodologia de acompanhamento 
desenvolvida, avaliar e recomendar melhorias a uma estrutura regional existente projetada especificamente para resposta de emergência de grande escala.

Zhang e Peng [30] aplicam a teoria da incerteza de forma a resolver o problema de localização de cobertura de instalações de serviços de emergência sob incerteza. Primeiramente, eles modelam o problema de cobertura do conjunto de instalação em um ambiente incerto, e, logo em seguida, dois modelos do problema de localização de cobertura máxima no mesmo ambiente. Os três são comparados e seus benefícios destacados, sendo aplicados posteriormente em um estudo de caso, de forma a demonstrar seus desempenhos.

Akbari, Eiselt e Pelot [33] realizam uma análise multicritério na localização de recursos de busca e resgaste marítimo. Os modelos de localização de cobertura máxima e da p-mediana são modificados e aplicados de acordo com as características dos problemas, resolvidos utilizando dados da região do oceano Atlântico do Canadá. As soluções ótimas são comparadas em cinco critérios de decisões. Em seguida, os resultados indicaram um aumento significativo na eficiência de utilização de recursos e disponibilidade de serviços com base no tempo de acesso e critérios de cobertura para as soluções fornecidas pelos modelos de otimização em comparação com a situação atual.

Smith et al. [36] abordam a aplicação prática de análise locacional para a localização de equipamentos de diagnóstico de HIV/AIDS em laboratórios da África do Sul, com o objetivo de encontrar um número apropriado do laboratório específico com locais eficientes.

\subsubsection{Centros de Distribuição}

Mohammadi, Jolai e Rostami [21] consideram um problema de rede de hub-andspoke com lotação ou congestionamento no sistema. Problemas de localização de hubs visam localizar as instalações e sua alocação da demanda dos nós. Os hubs são modelados como sistema de filas (M/M/c), sendo o modelo proposto aplicado em um sistema de transporte de carga, onde o número de caminhões segue uma distribuição de Poisson.

Mohammadi Jolai e Tavakkoli-Moghaddam [22] estendem o modelo original de problema de localização de $h u b$ ao considerar duas funções-objetivo: minimizar tanto os custos totais de investimentos correntes quanto o tempo máximo de transporte entre cada par origem-destino na rede.

Karimi e Bashiri [24] abordam problemas de localização de cobertura com diferentes tipos em redes de hub. O objetivo dos modelos propostos pelos autores é encontrar a localização dos hubs e alocar nós não hub aos nós de hub localizados, sujeitos ao tempo de viagem entre dois nós na origem-destino que não excedem um determinado limite. Posteriormente, esse modelo foi aplicado a um caso real da aviação iraniana.

Madani, Nookabadi e Hejazi [35] propõem um problema que visa selecionar hubs de backup para hubs indisponíveis e reencaminhar os fluxos relacionados, de forma a evitar interrupções em hubs ou indisponibilidade de rotas, pois as mesmas afetam o nível de serviços e geram custos excessivos.

\subsubsection{Design dos Nós}

Zarandi, Sisakht e Davari [23] propõem um projeto de rede de distribuição de cadeia de fornecimento de circuito fechado, em que um fluxo reverso é importado em um modelo de fluxo para frente. Esse modelo considera objetivos de coberturas para frente e reverso, e após isso, os resultados são comparados com um modelo que implementa os dois tipos de cobertura juntos. O principal objetivo é selecionar ótimas localizações, números e níveis de capacidade de plantas e armazéns.

Davari, Zarandi e Hemmati [26] utilizam variáveis difusas (tempo entre viagens) para lidar com incertezas, aplicando-as a uma versão difusa do problema de localização de 
cobertura máxima. Posteriormente, um modelo baseado na teoria da credibilidade e um algoritmo inteligente híbrido são utilizados para resolver o problema.

Jayalakshmi e Singh [31] propõem um algoritmo com o objetivo de localizar instalações de forma a maximizar a demanda total coberta. Diferente do problema de localização máxima de cobertura, que é considerado um modelo de cobertura individual, podendo gerar soluções de baixa qualidade, o CMCLP considera que todas as instalações cooperam no fornecimento de cobertura. A abordagem utilizada obteve melhores resultados comparados com outros modelos da literatura.

Bagherinejad e Shoeib [34] desenvolvem um modelo de problema de localização de cobertura máxima capacitado dinâmico, cujo objetivo é encontrar a localização ideal de um número de instalações em um horizonte de tempo de tal forma que, com a localização do número máximo de veículos no período $t$, a cobertura máxima é alcançada em todo o horizonte de tempo.

\subsubsection{Cliente}

Díaz et al. [32] visam maximizar a demanda de um conjunto de clientes por um conjunto de instalações localizado dentro de um conjunto de potenciais sites. Estes autores assumem que outros conjuntos de instalações de outras firmas existem e que os clientes escolhem livremente as instalações dentro de um dado raio de cobertura. O problema foi formulado de forma que o líder localize instalações de forma a maximizar a demanda coberta e o seguidor aloca os clientes para a instalação preferida entre aqueles selecionados pelo líder e as instalações de outras empresas.

\section{CONCLUSÃO}

A questão do problema de cobertura em localização de instalações é muito relevante para o mercado atual, principalmente na área de saúde, pois engloba questões como ambulâncias e serviço de combate a incêndio, e inclusive para operadoras de telefone e serviços similares. O trabalho envolveu 86 artigos publicados a partir de 2011 até 2018, que foram analisados e categorizados de acordo com a metodologia escolhida e temas mais abordados. Em relação à metodologia, foi revelado que a maioria dos artigos utilizaram trabalhos de modelagem ou estudo de caso. Nesta revisão, não foi possível achar artigos relacionados ao tema que usaram RSL como metodologia de pesquisa.

Para responder a PP-1, foi gerado um gráfico dos métodos utilizados pelos artigos, que foram combinação de algoritmos, comparação entre problemas e sob efeito de incerteza. Com o objetivo de responder a PP-2, os artigos foram classificados de acordo com os temas mais abordados: serviços de emergência, centros de distribuição, design de nós e cliente.

Na revisão, foi possível ver que diversos tópicos e áreas não são abordados, sendo importante o aprofundamento deste assunto em outras áreas. Sugere-se que, para trabalhos futuros, haja uma aplicação maior de revisão sistemática da literatura neste assunto, uma RSL com duas pessoas ou mais desse tópico e aprofundamento e aplicação de problemas de cobertura em localização de instalações em outras áreas.

\section{REFERÊNCIAS BIBLIOGRÁFICAS}

FARAHANI, R. Z.; HEKMATFAR, M. Facility location: concepts, models, algorithms and case studies. Physica-Verlag Heidelberg, Alemanha, p. 1-176, 2009.

SNYDER, L.V. Facility location under uncertainty: a review. IIE Transactions (Institute of Industrial Engineers), Reino Unido, v. 38, n. 7, p. 547-564, 2006. 
LU, Z.; BOSTEL, N. A facility location model for logistics systems including reverse flows: the case of remanufacturing activities. Computers and Operations Research, Reino Unido, v. 34, n. 2, p. 299-323, 2007.

BALCIK, B.; BEAMON, B. M. Facility location in humanitarian relief. International Journal of Logistics Research and Applications, Reino Unido, v. 11, n. 2, p. 101-121, 2008.

MELO, M.T.; NICKEL, S.; SALDANHA-DA-GAMA, F. Facility location and supply chain management: a review. European Journal of Operational Research, Holanda, v. 196, n. 2, p. 401-412, 2009.

FARAHANI, R.Z. et al. Covering problems in facility location: a review. Computers and Industrial Engineering, Reino Unido, v. 62, n. 1, p. 368-407, 2012.

OWEN, S.H.; DASKIN, M.S. Strategic facility location: a review. European Journal of Operational Research, Holanda, v. 111, n. 3, p. 423-447, 1998.

SCHILLING, D.A.; JAYARAMAN, V.; BARKHI, R. A review of covering problems in facility location. Location Science, Reino Unido, v. 1, n. 1, p. 25-55, 1993.

TOREGAS, C. et al. The location of emergency service facilities. Operations Research, Estados Unidos, v. 19, n. 6, p. 1363-1373, 1971.

MARÍN, A. et al. Multi-period stochastic covering location problems: Modeling framework and solution approach. European Journal of Operational Research, Holanda, v. 268, n. 2, p. 432-449, 2018.

CHURCH, R.; REVELLE, C. The maximal covering location problem. Papers of the Regional Science Association, Estados Unidos, v. 32, n. 1, p. 101-118, 1974.

LI, X. et al. Covering models and optimization techniques for emergency response facility location and planning: A review. Mathematical Methods of Operations Research, Alemanha, v. 74, n. 3, p. 281-310, 2011.

MCLAY, L.A. A maximum expected covering location model with two types of servers. IIE Transactions (Institute of Industrial Engineers), Reino Unido, v. 41, n. 8, p. 730-741, 2009.

ROUSSEAU, D.M.; MANNING, J.; DENYER, D. Evidence in management and organizational science: assembling the field's full weight of scientific knowledge through syntheses. The Academy of Management Annals, Reino Unido, v. 2, n. 1, p. 475-515, 2008.

DENYER, D.; TRANFIELD, D. Producing a systematic review. In: BUCHANAN, D.; BRYMAN, A. (Org.). The Sage Handbook of Organizational Research Methods. Londres: Sage Publications, 2009. p. 671-689.

THOMÉ, A.M.T.; SCAVARDA, L.F.; SCAVARDA, A.S. Conducting systematic literature review in operations management. Production Planning \& Control, Reino Unido, v. 27, n. 5, p. 408-420, 2016.

GLIGOR, D.M.F.; HOLCOMB, M.C. Understanding the role of logistics capabilities in achieving supply chain agility: a systematic literature review. Supply Chain Management, Reino Unido, v. 17, n. 4, p. 438-453. 2012.

MONGEON, P.; PAUL-HUS, A. The journal coverage of Web of Science and Scopus: a comparative analysis. Scientometrics, Holanda, v. 106, n. 1, p. 213-228, 2016. 
SEURING, S.; MÜLLER, M. From a literature review to a conceptual framework for sustainable supply chain management. Journal of Cleaner Production, Holanda, v. 16, n. 15, p. 1699-1710, 2008.

MURALI, P.; ORDÓÑEZ, F.; DESSOUKY, M.M. Facility location under demand uncertainty: response to a large-scale bio-terror attack. Socio-Economic Planning Sciences, Reino Unido, v. 46, n.1, p. 78-87, 2012.

MOHAMMADI, M.; JOLAI, F.; ROSTAMI, H. An M/M/c queue model for hub covering location problem. Mathematical and Computer Modelling, Reino Unido, v. 54, n. 11-12, p. 2623-2638, 2011.

MOHAMMADI, M.; JOLAI, F.; TAVAKKOLI-MOGHADDAM, R. Solving a new stochastic multi-mode p-hub covering location problem considering risk by a novel multiobjective algorithm. Applied Mathematical Modelling, Holanda, v. 33, n. 24, p. 1005310073, 2013.

ZARANDI, M.H.F.; SISAKHT, A.H.; DAVARI, S. Design of a closed-loop supply chain (CLSC) model using an interactive fuzzy goal programming. International Journal of Advanced Manufacturing Technology, Alemanha, v. 56, n. 5-8, p. 809-821, 2011.

KARIMI, H.; BASHIRI, M. Hub covering location problems with different coverage types. Scientia Iranica, Irã, v. 18, n. 6, p. 1571-1578, 2011.

KNIGHT, V.A.; HARPER, P.R.; SMITH, L. Ambulance allocation for maximal survival with heterogeneous outcome measures. Omega, Reino Unido, v. 40, n. 6, p. 918-926, 2012.

DAVARI, S.; ZARANDI, M. H. F; HEMMATI, A. Maximal covering location problem (MCLP) with fuzzy travel times. Expert Systems with Applications, Reino Unido, v. 38, n. 12, p. 14535-14541, 2011.

HE, S.Y.; KUO, Y.-H.; WU, D. Incorporating institutional and spatial factors in the selection of the optimal locations of public electric vehicle charging facilities: A case study of Beijing, China. Transportation Research Part C: Emerging Technologies, Reino Unido, v. 67, p. 131-148, 2016.

CHANTA, S.; MAYORGA, M.E.; MCLAY, L.A. Improving emergency service in rural areas: a bi-objective covering location model for EMS systems. Annals of Operations Research, Holanda, v. 221, n. 1, p. 133-159, 2014.

PAUL, N.R., LUNDAY, B.J., NURRE, S.G. A multiobjective, maximal conditional covering location problem applied to the relocation of hierarchical emergency response facilities. Omega, Reino Unido, v. 66, p. 147-158; 2017.

ZHANG, B.; PENG, J.; LI, S. Covering location problem of emergency service facilities in an uncertain environment. Applied Mathematical Modelling, Holanda, v. 51, p. 429-447, 2017.

JAYALAKSHMI, B.; SINGH, A. A hybrid artificial bee colony algorithm for the cooperative maximum covering location problem. International Journal of Machine Learning and Cybernetics, Estados Unidos, v. 8, n. 2, p. 69-697; 2017.

DÍAZ, J.A. et al. GRASP and hybrid GRASP-Tabu heuristics to solve a maximal covering location problem with customer preference ordering. Expert Systems with Applications, Reino Unido, v. 82, p. 67-76, 2017. 
AKBARI, A.; EISELT, H.A.; PELOT, R. A maritime search and rescue location analysis considering multiple criteria, with simulated demand. INFOR, Canadá, v. 56, n. 1, p. 92114, 2018.

BAGHERINEJAD, J.; SHOEIB, M. Dynamic capacitated maximal covering location problem by considering dynamic capacity. International Journal of Industrial Engineering Computations, Canadá, v. 9, n. 2, p. 249-264, 2018.

MADANI, S.R.; NOOKABADI, A. S.; HEJAZI, S. R. A bi-objective, reliable single allocation p-hub maximal covering location problem: Mathematical formulation and solution approach. Journal of Air Transport Management, Reino Unido, v. 68, p. 118-136, 2018.

SMITH, H.K. et al. Siting of HIV/AIDS diagnostic equipment in South Africa: a case study in locational analysis. International Transactions in Operational Research, Dinamarca, v. 25, n. 1, p, 39-336, 2018. 